\title{
Free Vibration of Embedded Porous Plate Using Third-Order Shear Deformation and Poroelasticity Theories
}

\author{
Ali Ghorbanpour Arani, Zahra Khoddami Maraghi, Mehdi Khani, and Iman Alinaghian \\ Faculty of Mechanical Engineering, University of Kashan, Kashan, Iran \\ Correspondence should be addressed to Ali Ghorbanpour Arani; aghorban@kashanu.ac.ir
}

Received 21 July 2016; Revised 17 October 2016; Accepted 7 November 2016; Published 24 January 2017

Academic Editor: Francis T. K. Au

Copyright ( 2017 Ali Ghorbanpour Arani et al. This is an open access article distributed under the Creative Commons Attribution License, which permits unrestricted use, distribution, and reproduction in any medium, provided the original work is properly cited.

\begin{abstract}
This research aims at studying free vibration of rectangular plate made of porous materials in which $Y$-foam, $G$-foam, and Coustone are used and compared with each other. To obtain the Biot formulation of the constitutive equations for a porous material, linear poroelasticity theory is used. Young modulus and density of porous plate are different in transverse direction versus porosity. In order to increase the accuracy of results in comparison with classical plate and first-order shear deformation theories, Reddy's theory was utilized in this research. Besides, five coupled equations of motion have been studied using Hamilton's principle and are solved by differential quadrature method (DQM). Detailed results of this study show the significant effect of aspect ratio, thickness ratio, boundary conditions, and porosity on dimensionless frequency and deflection of porous plate. Results of this study can contribute to the design of pneumatic conveying, handling, and control systems.
\end{abstract}

\section{Introduction}

Porous materials are materials which can be both organic and inorganic and contain pores (cavities, channels, and interstices) which are deeper than they are. These materials are mainly used in energy absorbing systems, porous electrodes, sound absorbers, filters, insulating materials, heat exchangers, construction materials, electromagnetic shielding, membrane, and membrane support [1].

This paper focuses on the vibration analysis of porous plate regarding its application in mechanical systems. In what follows, some of the works related to this issue are reviewed. Biot and Willis [2] studied the methods of measurement to determine the elastic coefficients based on the theory of the deformation of a porous elastic solid containing a compressible fluid. They also discussed the physical interpretation of the coefficients in various alternate forms. Mansutti et al. [3] presented steady flows of non-Newtonian fluids using a porous plate with suction or injection. In this study, the flow is the counterpart of the classical "asymptotic suction" problem, within the context of the non-Newtonian fluid models. Numerical solutions were compared with the solutions from a regular perturbation approach, as well as a singular perturbation. Leclaire et al. [4] presented the vibrations of a rectangular porous plate described by two coupled equations involving the time and space derivatives of the deflection and of the relative fluid-solid motion. Using Galerkin's variational method and classical theory of plates (nonporous), the equations were solved. The RayleighRitz decomposition on the basis of orthogonal or quasiorthogonal Eigen-functions was used and the solutions were inserted in the vibrational equations. Results of the study showed that if the viscoelastic damping of the solid is accounted, comparison between experimental and theoretical results on the plate defection is conveniently good. A simple model of the transverse vibrations of a thin rectangular porous plate saturated by a fluid was studied by Leclaire et al. [5]. The classical theory of homogeneous plates and Biot's stress-strain relations in an isotropic porous medium with a uniform porosity was used. They come to this conclusion that the resonance frequency is an increasing function of porosity and permeability and a decreasing function of tortuosity. A comparative study of Biot's theory and the linear theory of porous media for wave propagation problems was presented in Schanz and Diebels's article [6]. Regarding the results, a positive correlation between two approaches was 
observed in case of incompressible constituents, whereas in case of compressible constituents large differences appeared. Debowski and Magnucki [7] explored the dynamic stability of a porous rectangular plate to study an axial compressed porous-cellular rectangular plate which is a generalization of sandwich or multilayer plates. The result of this study shed light on the nonlinear hypothesis of deformation of the plane cross section for porous-cellular plate that includes the linear hypothesis for homogenous plate and the shear deformable effect. They proved that the mathematical model of the dynamic stability of the porous-cellular rectangular plate under pulsating compression load could be reduced to Mathieu's equation. Khorshidvand et al. [8] studied buckling analysis of radially solid circular plate made of porous material bounded with the layers of piezoelectric actuators. The results of their study showed that increasing the porosity decreases the critical load and the plate will be unstable. They also concluded that the plate with high porosity with the piezo layer has a better effect on stability of the plate in comparison to increasing the thickness of the porous plate. Reducing the porosity makes the behavior of the plate tend to incline to homogeneous/isotropic behavior. Jabbari et al. [9] investigated the buckling analysis of radially loaded solid circular plate made of porous material. Properties of their plate varied across the thickness. They derived the equilibrium and stability equations through the variational formulation based on Sander's nonlinear strain-displacement relation. In this study, the pores of the porous plate were saturated with a fluid. They come to the conclusion that increasing porosity decreases buckling load and the plate will be unstable by then. They also found that the Monotonous porosity is more unstable than symmetric and nonsymmetric porosity and the critical buckling load will be reduced by increasing the compressibility of fluid within the pores. An analysis of buckling of thin circular FG plates made of saturated porous-soft ferromagnetic materials in transverse magnetic field was done by Jabbari et al. [10]. The energy method based on the classical plate theory with the assumption of power-law composition for the constituent materials was used in the research. The research indicated that increasing the porosity makes the critical magnetic field decrease, the plate will be unstable, and the critical magnetic field will increase by increasing the thickness. Finally they concluded that Monotonous porosity is more unstable than the symmetric and nonsymmetric porosity in the aforementioned research. Joubaneh et al. [11] studied the thermal buckling analysis of solid circular plate made of porous material bounded with piezoelectric sensor-actuator patches in her research. The basic assumption under her research was that porous material properties vary with respect to thickness. The governing equations were obtained for clamped boundary condition based on mechanical nonlinear equilibrium and linear stability equations. The effects of porous plate's thickness, porosity, and piezoelectric thickness on thermal stability of the plate were investigated and studied in the research. A detailed analysis of complicated and general problem of a functionally graded (FG) porous variable thickness circular plate subjected to nonaxisymmetric and nonuniform shear and normal tractions was done by Behravan Rad and
Shariyat [12]. In their research, the plate was supported by a nonuniform Kerr elastic foundation based on the differential quadrature and state space vector techniques in the radial and thickness directions, respectively. The results showed that the induced magnetic quantities are mainly affected by the radial displacement component. They concluded that the material properties index, porosity, and the elastic foundation have positive effect and the compressibility parameter has an adverse and negative effect on the rigidity of the plate. Thermomechanical vibration characteristics of FG Reddy beams made of porous material subjected to various thermal loadings were investigated by Ebrahimi and Jafari [13]. They discussed the influences of several important parameters such as power-law exponents, porosity distributions, porosity volume fractions, and thermal effects on natural frequencies of the temperature-dependent FG beams.

Bending and vibration of FG beams were analyzed with a simple and refined trigonometric higher-order beam theory by Bourada et al. [14]. In this theory the thickness stretching effect $(\varepsilon z \neq 0)$ was also included and the transverse shear deformation effects were considered without requiring shear correction factors. In this paper the material properties varied in the thickness direction. Numerical results of the present theory were compared with other theories to show the effect of the inclusion of transverse normal strain on the deflections and stresses.

Bending and free vibration analysis of FG plates were developed using an original hyperbolic sine shear deformation theory by Neves et al. [15]. They used Carrera's Unified Formulation to derive the equations of motion for throughthe-thickness deformations. They showed the meshless technique in several numerical examples.

Hebali et al. [16] presented a new quasi-three-dimensional (3D) hyperbolic shear deformation theory for the bending and free vibration analysis of FG plates. This theory reduced the number of unknowns by dividing the transverse displacement into bending, shear, and thickness stretching parts. Also their theory satisfied the zero traction boundary conditions on the surfaces of the plate without using shear correction factor.

None of the aforementioned papers have investigated the vibration of porous plate using linear poroelasticity theory besides changing in Young modulus and density. The significance of this paper lies in using the third-order shear deformation theory (TSDT) to derive the equation of motions according to thickness ratio of plate for thin and thick plates. Variation of Young modulus and density in transvers direction of plate provides computational complexity that was solved by MAPLE and MATLAB software programs. Three different materials are compared with each other and different boundary conditions are applied on porous plate. The results show the effect of aspect ratio, thickness ratio, elastic medium, and porosity on dimensionless frequency of porous plate accordingly. This paper provides an initial platform for research on free and forced vibration of porous plates according to the many uses for this material. This article will help researchers to continue the studies about forced vibration of porous plate, drain and undrain states, 
nonconsolidated materials, and different states of fluid in porous materials.

Porous plate can be used in aerospace industry and nuclear reactor as a vibration damper, in sea structures, vessels, and submarines due to very low density and in reformer and catalysts due to the high specific surface. The results of this research can be applied in all cases.

\section{Structural Definition}

A schematic diagram of a porous plate is illustrated in Figure 1 in which geometrical parameters of length $a$, width $b$, and thickness $h$ are shown as well.

\section{Linear Poroelasticity Theory}

The Biot formulation of the constitutive equations for a fluidfilled porous material assumes the linearity between the stress and the strain and reversibility of the deformation process. Detournay and Chen [17] stated the linear poroelasticity theory of Biot in two statements:

(1) An increase of pore pressure induces a dilation of pore.

(2) Compression of the pore causes an increase in pore pressure.

According to the above-mentioned assumptions, stress-strain relation for porous material is shown in $[8,9]$

$$
\sigma_{i j}=\bar{C}_{i j} \varepsilon_{i j}-P_{p} \alpha \delta_{i j}
$$

in which

$$
\begin{aligned}
& {\left[\begin{array}{c}
\sigma_{x x} \\
\sigma_{y y} \\
\sigma_{y z} \\
\sigma_{x z} \\
\sigma_{x y}
\end{array}\right]} \\
& =\left[\begin{array}{ccccc}
\bar{C}_{11}-M \alpha^{2} & \bar{C}_{12} & 0 & 0 & 0 \\
\bar{C}_{21} & \bar{C}_{22}-M \alpha^{2} & 0 & 0 & 0 \\
0 & 0 & \bar{C}_{44} & 0 & 0 \\
0 & 0 & 0 & \bar{C}_{55} & 0 \\
0 & 0 & 0 & 0 & \bar{C}_{66}
\end{array}\right]\left[\begin{array}{c}
\varepsilon_{x x} \\
\varepsilon_{y y} \\
\varepsilon_{y z} \\
\varepsilon_{x z} \\
\varepsilon_{x y}
\end{array}\right],
\end{aligned}
$$

where $\sigma_{i j}$ and $\varepsilon_{i j}$ are normal and shear stresses and the terms of engineering constant are as follows [18]:

$$
\begin{aligned}
& \bar{C}_{11}=\frac{E(z)}{\left(1-v^{2}\right)} ; \\
& \bar{C}_{12}=\bar{C}_{21}=\frac{v E(z)}{\left(1-v^{2}\right)} ; \\
& \bar{C}_{44}=\bar{C}_{55}=\bar{C}_{66}=\frac{E(z)}{(1+v)}
\end{aligned}
$$

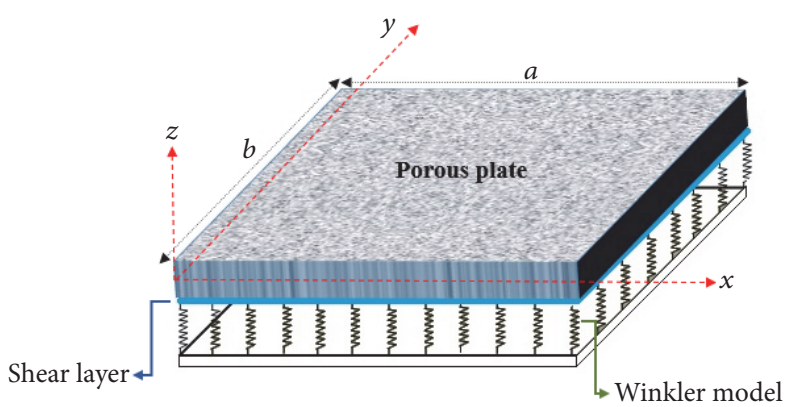

FIgURE 1: A schematic diagram of a porous plate.

in which $E$ and $v$ are Young modulus and Poisson's ratio, respectively.

(i) The constant $M$ is sometimes called the Biot modulus. The constant $\alpha$ is known as the ratio of the fluid volume gained (or lost) in a material element to the volume change of that element, when the pore pressure is allowed to return to its initial state and it varies between 0 and 1 . These parameters are introduced as follows $[4,17]$ :

$$
P_{p}=-M \alpha \varepsilon_{k k} ; \quad \alpha=1-c(1-\phi) .
$$

(ii) Coefficient $c$ can be regarded as a coefficient which characterizes the consolidation state of the heterogeneous material. $c$ is between 0 for nonconsolidated materials and 1 for consolidated materials [4]; here $c=1$.

(iii) Porosity $(\phi)$ is defined as ratio of volume of pore space to the volume of porous material. Change in the compressibility of the fluid or porosity affects the bulk constants [17].

The variation of Young modulus and density in transverse direction of porous plate is shown as follows $[10,11]$ :

$$
\begin{aligned}
& E(z)=E_{0}\left[1-e_{1} \cos \left(\left(\frac{\pi}{2 h}\right)\left(z+\frac{h}{2}\right)\right)\right], \\
& \rho(z)=\rho_{0}\left[1-e_{1} \cos \left(\left(\frac{\pi}{2 h}\right)\left(z+\frac{h}{2}\right)\right)\right] .
\end{aligned}
$$

In this equation, $e_{1}$ is the coefficient of plate porosity, $E_{1}$ and $E_{0}$ are Young's modulus of elasticity at $z=h / 2$ and $z=-h / 2$, and $\rho_{1}$ and $\rho_{0}$ are the density at $z=h / 2$ and $z=-h / 2$, respectively. $G=E /(1+2 v)$ is the relationship between the modulus of elasticity and shear modulus and $v$ is Poisson's ratio, which is assumed to be constant across the plate thickness. Mechanical properties of the porous material vary across the thickness of the plate $e_{1}=1-G_{1} / G_{0}=$ $1-E_{1} / E_{0}$, in which $0 \leq e_{1} \leq 1$.

Simplified expressions can be extracted for the poroelastic parameters for limiting cases in particular [17]. 
For highly compressible fluid constituent $\left(K_{f} / K \ll\right.$ 1, $f$ : fluid), the approximate expressions for $M$ are

$$
\begin{aligned}
& M=\frac{K_{f}}{\phi}, \\
& K=\frac{E}{3(1-2 v)} .
\end{aligned}
$$

In the limit, $K_{f} \rightarrow 0$ and $M \rightarrow 0$. In other words, the porous material behaves as an elastic material without fluid where $\phi$ is called porosity and $K$ is bulk modulus.

\section{Third-Order Shear Deformation Theory (TSDT)}

TSDT expands the first-order shear deformation theory (FSDT) by assuming the following:

(i) Shear strain and consequently shear stress are not constant through the plate thickness, where TSDT leads to better results for a moderately thick plate.

(ii) A shear correction factor is not required for the strain equations.

(iii) The displacement field accommodates a quadratic variation of the transverse shear through the thickness and the vanishing of transverse shear is mainly highlighted on the top and bottom surfaces of the plate $[18,19]$.

The displacement field of TSDT for plates is presented here [5]:

$$
\begin{aligned}
& \widetilde{U}(x, y, z, t) \\
&= u_{0}(x, y, t)+z \theta_{1}(x, y, t) \\
&-\frac{4}{3} \frac{z^{3}}{h^{2}}\left(\theta_{1}(x, y, t)+\frac{\partial}{\partial x} w_{0}(x, y, t)\right), \\
& \widetilde{V}(x, y, z, t) \quad v_{0}(x, y, t)+z \theta_{2}(x, y, t) \\
& \quad-\frac{4}{3} \frac{z^{3}}{h^{2}}\left(\theta_{2}(x, y, t)+\frac{\partial}{\partial y} w_{0}(x, y, t)\right), \\
& \widetilde{W}(x, y, z, t)=w_{0}(x, y, t),
\end{aligned}
$$

where $u_{0}(x, y, t), v_{0}(x, y, t), w_{0}(x, y, t)$ are displacement functions along $(x, y, z)$ directions and $\theta_{1}(x, y, t), \theta_{2}(x, y, t)$ are rotations about $x$ - and $y$-axes and $t$ is time. Using Hooke's law, the linear strain field for TSDT is being calculated.

\section{Energy Method in Porous Plate}

In this part, the energy method is used to work out the governing equations. Strain and kinetic energy of the rectangular porous plate is calculated and presented $[20,21]$ :

$$
\begin{aligned}
U= & \frac{1}{2} \\
& \cdot \int_{V}\left(\sigma_{x x} \varepsilon_{x x}+\sigma_{y y} \varepsilon_{y y}+\tau_{x y} \gamma_{x y}+\tau_{x z} \gamma_{x z}+\tau_{y z} \gamma_{y z}\right) d V, \\
K= & \frac{1}{2}(\rho h) \\
& \cdot\left(\int_{0}^{b} \int_{0}^{a}\left[\left(\frac{\partial \widetilde{U}}{\partial t}\right)^{2}+\left(\frac{\partial \widetilde{V}}{\partial t}\right)^{2}+\left(\frac{\partial \widetilde{W}}{\partial t}\right)^{2}\right] d x d y\right) .
\end{aligned}
$$

Using (1) and (7), the strain energy of porous plate can be derived.

$\rho$ is the density of porous plate and $\tau_{i j}$ is tractions. By substituting (7) into (9), the kinetic energy is obtained.

5.1. External Work. Pasternak foundation is able to consider transverse shear loads and normal loads. The effect of surrounding elastic medium on the porous plate which is simulated with Pasternak model is considered as follows [21]:

$$
F_{\mathrm{Ex}}=K_{w} \widetilde{W}-K_{G}\left(\frac{\partial^{2} \widetilde{W}}{\partial x^{2}}+\frac{\partial^{2} \widetilde{W}}{\partial y^{2}}\right),
$$

where $K_{w}$ and $K_{G}$ are Winkler modulus for normal load and shear modulus for transverse shear loads, respectively. Therefore, the external work due to elastic medium is calculated as

$$
\Sigma=\frac{1}{2} \int_{0}^{b} \int_{0}^{a} F_{\mathrm{Ex}} \widetilde{W} d x d y
$$

5.2. Hamilton's Principle. Hamilton's principle is utilized to obtain equations of motion. The principle can be stated in an analytical form in which the first variation form of equations must be zero [20].

$$
\delta \int_{t_{1}}^{t_{2}}[U-(K+\Sigma)] d t=0 .
$$

In this equation, $\delta U, \delta K$, and $\delta \Sigma$ are variations of strain energy, variation of Kinetic energy, and variation of external work. We substituted (8) and (9) and external work into (12) for TSDT and used dimensionless parameters afterwards which were introduced in

$$
\begin{aligned}
(\zeta, \eta) & =\left(\frac{x}{a}, \frac{y}{b}\right), \\
(U, V, W) & =\left(\frac{u_{0}}{a}, \frac{v_{0}}{b}, \frac{w_{0}}{h}\right), \\
\gamma & =\frac{a}{b} \\
C_{i j} & =\frac{\bar{C}_{i j}}{E},
\end{aligned}
$$




$$
\begin{aligned}
\left(\alpha_{c}, \beta_{c}\right) & =\left(\frac{h}{a}, \frac{h}{b}\right), \\
k_{w} & =\frac{K_{w} h}{E}, \\
k_{g} & =\frac{K_{G}}{a \cdot E}, \\
\tau & =\frac{t}{a} \sqrt{\frac{E}{\rho}}, \\
\text { Ipdi } & =\frac{I p i}{h^{i+1}} \quad(i=2,4,6), \\
\text { Idi } & =\frac{I i}{h^{i+1}} .
\end{aligned}
$$

The equations of motion are worked out by setting the coefficients $\delta U, \delta V, \delta W, \delta \theta_{1}, \delta \theta_{2}$ equal to zero:

$$
\begin{aligned}
& \delta U=-\frac{1}{2} I_{0} \gamma \beta_{c} C_{44} \frac{\partial^{2}}{\partial \eta^{2}} U+\frac{2}{3} \beta_{c}{ }^{2} C_{44} I_{3} \frac{\partial^{2}}{\partial \eta^{2}} \theta_{1} \\
& -\alpha_{c}{ }^{2} C_{11} I_{1} \frac{\partial^{2}}{\partial \zeta^{2}} \theta_{1}+\frac{2}{3} \beta_{c}^{2} \alpha_{c} C_{21} I_{3} \frac{\partial^{3}}{\partial \eta^{2} \partial \zeta} W \\
& +\frac{2}{3} \beta_{c} \alpha_{c} C_{21} I_{3} \frac{\partial^{2}}{\partial \eta \partial \zeta} \theta_{2}+\frac{4}{3} \alpha_{c}^{3} C_{11} I_{3} \frac{\partial^{3}}{\partial \zeta^{3}} W \\
& +\frac{2}{3} \beta_{c} \alpha_{c} C_{44} I_{3} \frac{\partial^{2}}{\partial \eta \partial \zeta} \theta_{2} \\
& +\frac{2}{3} \beta_{c}{ }^{2} \alpha_{c} C_{12} I_{3} \frac{\partial^{3}}{\partial \eta^{2} \partial \zeta} W \\
& +\frac{4}{3} \beta_{c}{ }^{2} \alpha_{c} C_{44} I_{3} \frac{\partial^{3}}{\partial \eta^{2} \partial \zeta} W \\
& +\frac{2}{3} \beta_{c} \alpha_{c} C_{12} I_{3} \frac{\partial^{2}}{\partial \eta \partial \zeta} \theta_{2}-\frac{1}{2} \beta_{c}{ }^{2} C_{44} I_{1} \frac{\partial^{2}}{\partial \eta^{2}} \theta_{1} \\
& +\frac{4}{3} \alpha_{c}^{2} C_{11} I_{3} \frac{\partial^{2}}{\partial \zeta^{2}} \theta_{1}-\frac{1}{2} I_{0} \alpha_{c} C_{21} \frac{\partial^{2}}{\partial \eta \partial \zeta} V \\
& -I_{0} \alpha_{c} C_{11} \frac{\partial^{2}}{\partial \zeta^{2}} U-\frac{1}{2} \beta_{c} \alpha_{c} C_{44} I_{1} \frac{\partial^{2}}{\partial \eta \partial \zeta} \theta_{2} \\
& -\frac{1}{2} \beta_{c} \alpha_{c} C_{12} I_{1} \frac{\partial^{2}}{\partial \eta \partial \zeta} \theta_{2}-\frac{1}{2} \beta_{c} \alpha_{c} C_{21} I_{1} \frac{\partial^{2}}{\partial \eta \partial \zeta} \theta_{2} \\
& -\frac{1}{2} I_{0} \alpha_{c} C_{44} \frac{\partial^{2}}{\partial \eta \partial \zeta} V-\frac{1}{2} I_{0} \alpha_{c} C_{12} \frac{\partial^{2}}{\partial \eta \partial \zeta} V \\
& -M \alpha^{2} \alpha_{c} \frac{\partial^{2}}{\partial \eta \partial \zeta} V-M \alpha^{2} \alpha_{c} \frac{\partial^{2}}{\partial \zeta^{2}} U+I_{0} \alpha_{c} \frac{\partial^{2}}{\partial \tau^{2}} U \\
& +\alpha_{c}^{2} I_{1} \frac{\partial^{2}}{\partial \tau^{2}} \theta_{1}-\frac{4}{3} \alpha_{c}^{2} I_{3} \frac{\partial^{2}}{\partial \tau^{2}} \theta_{1} \\
& -\frac{4}{3} \alpha_{c}^{3} I_{3} \frac{\partial^{3}}{\partial \tau^{2} \partial \zeta} W=0
\end{aligned}
$$

$$
\begin{aligned}
& \delta V=-\frac{1}{2 \gamma} I_{0} \alpha_{c} C_{44} \frac{\partial^{2}}{\partial \zeta^{2}} V+\frac{2}{3} \alpha_{c}^{2} \beta_{c} C_{12} I_{3} \frac{\partial^{3}}{\partial \eta \partial \zeta^{2}} W \\
& +\frac{4}{3} \beta_{c}{ }^{3} C_{22} I_{3} \frac{\partial^{3}}{\partial \eta^{3}} W+\frac{2}{3} \alpha_{c}^{2} \beta_{c} C_{21} I_{3} \frac{\partial^{3}}{\partial \eta \partial \zeta^{2}} W \\
& -\frac{2}{3} \alpha_{c} \beta_{c} C_{21} I_{3} \frac{\partial^{2}}{\partial \eta \partial \zeta} \theta_{1}+\frac{2}{3} \alpha_{c} \beta_{c} C_{12} I_{3} \frac{\partial^{2}}{\partial \eta \partial \zeta} \theta_{1} \\
& +\frac{4}{3} \alpha_{c}^{2} \beta_{c} C_{44} I_{3} \frac{\partial^{3}}{\partial \eta \partial \zeta^{2}} W \\
& +\frac{2}{3} \alpha_{c} \beta_{c} C_{44} I_{3} \frac{\partial^{2}}{\partial \eta \partial \zeta} \theta_{1}-\frac{1}{2} \alpha_{c}^{2} C_{44} I_{1} \frac{\partial^{2}}{\partial \zeta^{2}} \theta_{2} \\
& -C_{22} \beta_{c}^{2} I_{1} \frac{\partial^{2}}{\partial \eta^{2}} \theta_{2}+\frac{2}{3} \alpha_{c}{ }^{2} C_{44} I_{3} \frac{\partial^{2}}{\partial \zeta^{2}} \theta_{2} \\
& -\frac{1}{2} I_{0} \alpha_{c} \beta_{c} C_{21} \frac{\partial^{2}}{\partial \eta \partial \zeta} U+\frac{4}{3} \beta_{c}{ }^{2} C_{22} I_{3} \frac{\partial^{2}}{\partial \eta^{2}} \theta_{2} \\
& -\frac{1}{2} \alpha_{c} \beta_{c} C_{12} I_{1} \frac{\partial^{2}}{\partial \eta \partial \zeta} \theta_{1}-\frac{1}{2} \alpha_{c} \beta_{c} C_{44} I_{1} \frac{\partial^{2}}{\partial \eta \partial \zeta} \theta_{1} \\
& -\frac{1}{2} \alpha_{c} \beta_{c} C_{21} I_{1} \frac{\partial^{2}}{\partial \eta \partial \zeta} \theta_{1}-I_{0} \beta_{c} C_{22} \frac{\partial^{2}}{\partial \eta^{2}} V \\
& -\frac{1}{2} I_{0} \beta_{c} C_{44} \frac{\partial^{2}}{\partial \eta \partial \zeta} U-\frac{1}{2} I_{0} \beta_{c} C_{12} \frac{\partial^{2}}{\partial \eta \partial \zeta} U \\
& -M \alpha^{2} \beta_{c} \frac{\partial^{2}}{\partial \eta \partial \zeta} U-M \alpha^{2} \beta_{c} \frac{\partial^{2}}{\partial \eta^{2}} V \\
& +\frac{\alpha_{c}}{\gamma} I_{0} \frac{\partial^{2}}{\partial \tau^{2}} V+\alpha_{c}^{2} I_{1} \frac{\partial^{2}}{\partial \tau^{2}} \theta_{2}-\frac{4}{3} \alpha_{c}^{2} I_{3} \frac{\partial^{2}}{\partial \tau^{2}} \theta_{2} \\
& -\frac{4}{3} \alpha_{c}^{2} \beta_{c} I_{3} \frac{\partial^{3}}{\partial \tau^{2} \partial \eta} W=0, \\
& \delta W=\frac{8}{9} \alpha_{c}^{2} \beta_{c} C_{21} I_{6} \frac{\partial^{3}}{\partial \eta \partial \zeta^{2}} \theta_{2} \\
& -\frac{2}{3} \alpha_{c}^{2} \beta_{c} C_{12} I_{4} \frac{\partial^{3}}{\partial \eta \partial \zeta^{2}} \theta_{2} \\
& +\frac{16}{9} \alpha_{c}^{2} \beta_{c}^{2} C_{12} I_{6} \frac{\partial^{4}}{\partial \eta^{2} \partial \zeta^{2}} W \\
& +\frac{16}{9} \alpha_{c}^{2} \beta_{c}^{2} C_{21} I_{6} \frac{\partial^{4}}{\partial \eta^{2} \partial \zeta^{2}} W \\
& +\frac{32}{9} \alpha_{c}^{2} \beta_{c}^{2} C_{44} I_{6} \frac{\partial^{4}}{\partial \eta^{2} \partial \zeta^{2}} W \\
& +\frac{16}{9} \alpha_{c}^{2} \beta_{c} C_{44} I_{6} \frac{\partial^{3}}{\partial \eta \partial \zeta^{2}} \theta_{2} \\
& +\frac{8}{9} \alpha_{c} \beta_{c}{ }^{2} C_{21} I_{6} \frac{\partial^{3}}{\partial \eta^{2} \partial \zeta} \theta_{1}
\end{aligned}
$$




$$
\begin{aligned}
& -\frac{2}{3} \alpha_{c} \beta_{c}{ }^{2} C_{21} I_{4} \frac{\partial^{3}}{\partial \eta^{2} \partial \zeta} \theta_{1} \\
& -\frac{2}{3} \alpha_{c}{ }^{2} C_{21} I_{3} \frac{\partial^{3}}{\partial \eta \partial \zeta^{2}} V \frac{16}{9} M \alpha^{2} I_{p 6} \beta_{c}{ }^{4} \frac{\partial^{4}}{\partial \eta^{4}} W \\
& -\frac{2}{3} \alpha_{c} \beta_{c}^{2} C_{12} I_{4} \frac{\partial^{3}}{\partial \eta^{2} \partial \zeta} \theta_{1} \\
& +\frac{16}{9} M \alpha^{2} I_{p 6} \alpha_{c}{ }^{4} \frac{\partial^{4}}{\partial \zeta^{4}} W \\
& +\frac{8}{9} \alpha_{c} \beta_{c}^{2} C_{12} I_{6} \frac{\partial^{3}}{\partial \eta^{2} \partial \zeta} \theta_{1} \\
& +\frac{16}{9} M \alpha^{2} I_{p 6} \beta_{c}^{2} \alpha_{c} \frac{\partial^{3}}{\partial \eta^{2} \partial \zeta} \theta_{1} \\
& -\frac{2}{3} \alpha_{c}^{2} \beta_{c} C_{21} I_{4} \frac{\partial^{3}}{\partial \eta \partial \zeta^{2}} \theta_{2} \\
& +\frac{16}{9} \alpha_{c} \beta_{c}{ }^{2} C_{44} I_{6} \frac{\partial^{3}}{\partial \eta^{2} \partial \zeta} \theta_{1} \\
& -\frac{4}{3} \alpha_{c}^{2} \beta_{c} C_{44} I_{4} \frac{\partial^{3}}{\partial \eta \partial \zeta^{2}} \theta_{2} \\
& -\frac{4}{3} \alpha_{c} \beta_{c}{ }^{2} C_{44} I_{4} \frac{\partial^{3}}{\partial \eta^{2} \partial \zeta} \theta_{1}-\frac{1}{2} I_{0} \alpha_{c}{ }^{2} C_{55} \frac{\partial^{2}}{\partial \zeta^{2}} W \\
& -\frac{1}{2} I_{0} \alpha_{c} C_{55} \frac{\partial}{\partial \zeta} \theta_{1}-\frac{1}{2} I_{0} \beta_{c}^{2} C_{66} \frac{\partial^{2}}{\partial \eta^{2}} W \\
& -\frac{1}{2} I_{0} \beta_{c} C_{66} \frac{\partial}{\partial \eta} \theta_{2}-8 \alpha_{c} C_{55} I_{4} \frac{\partial}{\partial \zeta} \theta_{1} \\
& +4 \alpha_{c} C_{55} I_{2} \frac{\partial}{\partial \zeta} \theta_{1}+4 \beta_{c} C_{66} I_{2} \frac{\partial}{\partial \eta} \theta_{2} \\
& -8 \beta_{c} C_{66} I_{4} \frac{\partial}{\partial \eta} \theta_{2}-\frac{4}{3} \alpha_{c}^{3} C_{11} I_{4} \frac{\partial^{3}}{\partial \zeta^{3}} \theta_{1} \\
& +\frac{8}{9} \alpha_{c}^{2} \beta_{c} C_{12} I_{6} \frac{\partial^{3}}{\partial \eta \partial \zeta^{2}} \theta_{2} \\
& -\frac{2}{3} \beta_{c}{ }^{2} C_{21} I_{3} \frac{\partial^{3}}{\partial \eta^{2} \partial \zeta} U-\frac{2}{3} C_{12} \alpha_{c}{ }^{2} I_{3} \frac{\partial^{3}}{\partial \eta \partial \zeta^{2}} V \\
& -\frac{4}{3} \alpha_{c}{ }^{2} C_{11} I_{3} \frac{\partial^{3}}{\partial \zeta^{3}} U-\frac{4}{3} \beta_{c}^{2} C_{22} I_{3} \frac{\partial^{3}}{\partial \eta^{3}} V \\
& +\frac{16}{9} \alpha_{c}{ }^{4} C_{11} I_{6} \frac{\partial^{4}}{\partial \zeta^{4}} W+\frac{16}{9} \beta_{c}{ }^{4} C_{22} I_{6} \frac{\partial^{4}}{\partial \eta^{4}} W \\
& -8 \beta_{c}{ }^{2} C_{66} I_{4} \frac{\partial^{2}}{\partial \eta^{2}} W+4 \beta_{c}{ }^{2} C_{66} I_{2} \frac{\partial^{2}}{\partial \eta^{2}} W \\
& +8 \alpha_{c}^{2} C_{55} I_{4} \frac{\partial^{2}}{\partial \zeta^{2}} W+4 \alpha_{c}^{2} C_{55} I_{2} \frac{\partial^{2}}{\partial \zeta^{2}} W \\
& +\frac{16}{9} \beta_{c}{ }^{3} C_{22} I_{6} \frac{\partial^{3}}{\partial \eta^{3}} \theta_{2}-\frac{4}{3} \beta_{c}{ }^{2} C_{44} I_{3} \frac{\partial^{3}}{\partial \eta^{2} \partial \zeta} U \\
& +\frac{16}{9} \alpha_{c}{ }^{3} C_{11} I_{6} \frac{\partial^{3}}{\partial \zeta^{3}} \theta_{1}-\frac{2}{3} \beta_{c}{ }^{2} C_{12} I_{3} \frac{\partial^{3}}{\partial \eta^{2} \partial \zeta} U \\
& -\frac{4}{3} \alpha_{c}{ }^{2} C_{44} I_{3} \frac{\partial^{3}}{\partial \eta \partial \zeta^{2}} V-\frac{4}{3} \beta_{c}{ }^{3} C_{22} I_{4} \frac{\partial^{3}}{\partial \eta^{3}} \theta_{2} \\
& +\frac{16}{9} M \alpha^{2} I_{p 6} \beta_{c} \alpha_{c}^{2} \frac{\partial^{3}}{\partial \eta \partial \zeta^{2}} \theta_{2} \\
& +\frac{32}{9} M \alpha^{2} I_{p 6} \beta_{c}^{2} \alpha_{c}^{2} \frac{\partial^{4}}{\partial \eta^{2} \partial \zeta^{2}} W \\
& +\frac{16}{9} M \alpha^{2} I_{p 6} \beta_{c}^{3} \frac{\partial^{3}}{\partial \eta^{3}} \theta_{2} \\
& +\frac{16}{9} M \alpha^{2} I_{p 6} \alpha_{c}{ }^{3} \frac{\partial^{3}}{\partial \zeta^{3}} \theta_{1} \\
& -\frac{4}{3} M \alpha^{2} I_{p 4} \beta_{c} \alpha_{c}^{2} \frac{\partial^{3}}{\partial \eta \partial \zeta^{2}} \theta_{2} \\
& -\frac{4}{3} M \alpha^{2} I_{p 4} \alpha_{c}^{3} \frac{\partial^{3}}{\partial \zeta^{3}} \theta_{1} \\
& -\frac{4}{3} M \alpha^{2} I_{p 4} \beta_{c}^{2} \alpha_{c} \frac{\partial^{3}}{\partial \eta^{2} \partial \zeta} \theta_{1} \\
& -\frac{4}{3} M \alpha^{2} I_{p 4} \beta_{c}{ }^{3} \frac{\partial^{3}}{\partial \eta^{3}} \theta_{2}+\frac{4}{3} \alpha_{c}{ }^{2} I_{3} \frac{\partial^{3}}{\partial \tau^{2} \partial \zeta} U \\
& +\frac{4}{3} \alpha_{c}^{3} I_{4} \frac{\partial^{3}}{\partial \tau^{2} \partial \zeta} \theta_{1}-\frac{16}{9} \alpha_{c}^{3} I_{6} \frac{\partial^{3}}{\partial \tau^{2} \partial \zeta} \theta_{1} \\
& -\frac{16}{9} \alpha_{c}^{4} I_{6} \frac{\partial^{4}}{\partial \tau^{2} \partial \zeta^{2}} W-\frac{4}{3} \alpha_{c}^{2} I_{3} \frac{\partial^{3}}{\partial \tau^{2} \partial \eta} V \\
& +\frac{4}{3} \alpha_{c}^{2} \beta_{c} I_{4} \frac{\partial^{3}}{\partial \tau^{2} \partial \eta} \theta_{2}-\frac{16}{9} \alpha_{c}^{2} \beta_{c} I_{6} \frac{\partial^{3}}{\partial \tau^{2} \partial \eta} \theta_{2} \\
& -\frac{16}{9} \alpha_{c}^{2} \beta_{c}^{2} I_{6} \frac{\partial^{4}}{\partial \tau^{2} \partial \eta^{2}} W+\alpha_{c}{ }^{2} I_{0} \frac{\partial^{2}}{\partial \tau^{2}} W=0, \\
& \delta \theta_{1}=\frac{1}{2} I_{0} \alpha_{c} C_{55} \frac{\partial}{\partial \zeta} W-4 C_{55} I_{2} \theta_{1}+8 C_{55} I_{4} \theta_{1} \\
& +\frac{2}{3} \beta_{c}^{2} \alpha_{c} C_{21} I_{4} \frac{\partial^{3}}{\partial \eta^{2} \partial \zeta} W \\
& -\frac{8}{9} \beta_{c}^{2} \alpha_{c} C_{21} I_{6} \frac{\partial^{3}}{\partial \eta^{2} \partial \zeta} W \\
& -\frac{16}{9} \beta_{c}^{2} \alpha_{c} C_{44} I_{6} \frac{\partial^{3}}{\partial \eta^{2} \partial \zeta} W \\
& -\frac{8}{9} \beta_{c} \alpha_{c} C_{12} I_{6} \frac{\partial^{2}}{\partial \eta \partial \zeta} \theta_{2}+\frac{4}{3} \beta_{c} \alpha_{c} C_{12} I_{4} \frac{\partial^{2}}{\partial \eta \partial \zeta} \theta_{2}
\end{aligned}
$$




$$
\begin{aligned}
& -\frac{8}{9} \beta_{c} \alpha_{c} C_{44} I_{6} \frac{\partial^{2}}{\partial \eta \partial \zeta} \theta_{2}+\frac{4}{3} \beta_{c} \alpha_{c} C_{44} I_{4} \frac{\partial^{2}}{\partial \eta \partial \zeta} \theta_{2} \\
& -\frac{16}{9} M \alpha^{2} I_{p 6} \beta_{c}^{2} \alpha_{c} \frac{\partial^{3}}{\partial \eta^{2} \partial \zeta} W \\
& +\frac{2}{3} \beta_{c}^{2} \alpha_{c} C_{12} I_{4} \frac{\partial^{3}}{\partial \eta^{2} \partial \zeta} W \\
& -\frac{8}{9} \beta_{c}{ }^{2} \alpha_{c} C_{12} I_{6} \frac{\partial^{3}}{\partial \eta^{2} \partial \zeta} W \\
& +\frac{4}{3} \beta_{c} \alpha_{c} C_{21} I_{4} \frac{\partial^{2}}{\partial \eta \partial \zeta} \theta_{2} \\
& +\frac{4}{3} \beta_{c}^{2} \alpha_{c} C_{44} I_{4} \frac{\partial^{3}}{\partial \eta^{2} \partial \zeta} W \\
& -\frac{8}{9} \beta_{c} \alpha_{c} C_{21} I_{6} \frac{\partial^{2}}{\partial \eta \partial \zeta} \theta_{2}+\frac{2}{3} \beta_{c} \gamma C_{44} I_{3} \frac{\partial^{2}}{\partial \eta^{2}} U \\
& -\frac{1}{2} \beta_{c} \gamma C_{44} I_{1} \frac{\partial^{2}}{\partial \eta^{2}} U-\frac{1}{2} \beta_{c} \alpha_{c} C_{44} I_{2} \frac{\partial^{2}}{\partial \eta \partial \zeta} \theta_{2} \\
& -\frac{1}{2} \beta_{c} \alpha_{c} C_{12} I_{2} \frac{\partial^{2}}{\partial \eta \partial \zeta} \theta_{2}-\frac{1}{2} \beta_{c} \alpha_{c} C_{21} I_{2} \frac{\partial^{2}}{\partial \eta \partial \zeta} \theta_{2} \\
& -\alpha_{c}^{2} C_{11} I_{2} \frac{\partial^{2}}{\partial \zeta^{2}} \theta_{1}-\alpha_{c} C_{11} I_{1} \frac{\partial^{2}}{\partial \zeta^{2}} U \\
& +\frac{8}{3} \alpha_{c}^{2} C_{11} I_{4} \frac{\partial^{2}}{\partial \zeta^{2}} \theta_{1}-\frac{16}{9} \alpha_{c}^{2} C_{11} I_{6} \frac{\partial^{2}}{\partial \zeta^{2}} \theta_{1} \\
& +\frac{2}{3} \alpha_{c} C_{44} I_{3} \frac{\partial^{2}}{\partial \eta \partial \zeta} V+\frac{4}{3} \alpha_{c} C_{11} I_{3} \frac{\partial^{2}}{\partial \zeta^{2}} U \\
& -\frac{8}{9} \beta_{c}{ }^{2} C_{44} I_{6} \frac{\partial^{2}}{\partial \eta^{2}} \theta_{1}+\frac{4}{3} \beta_{c}{ }^{2} C_{44} I_{4} \frac{\partial^{2}}{\partial \eta^{2}} \theta_{1} \\
& -\frac{1}{2} \beta_{c}{ }^{2} C_{44} I_{2} \frac{\partial^{2}}{\partial \eta^{2}} \theta_{1}-\frac{16}{9} \alpha_{c}{ }^{3} C_{11} I_{6} \frac{\partial^{3}}{\partial \zeta^{3}} W \\
& +\frac{4}{3} \alpha_{c}{ }^{3} C_{11} I_{4} \frac{\partial^{3}}{\partial \zeta^{3}} W-\frac{1}{2} \alpha_{c} C_{12} I_{1} \frac{\partial^{2}}{\partial \eta \partial \zeta} V \\
& +\frac{2}{3} \alpha_{c} C_{21}{ }^{2} I_{3} \frac{\partial^{2}}{\partial \eta \partial \zeta} V-4 \alpha_{c} C_{55} I_{2} \frac{\partial}{\partial \zeta} W \\
& +8 \alpha_{c} C_{55} I_{4} \frac{\partial}{\partial \zeta} W+\frac{2}{3} \alpha_{c} C_{12} I_{3} \frac{\partial^{2}}{\partial \eta \partial \zeta} V \\
& -\frac{1}{2} \alpha_{c} C_{44} I_{1} \frac{\partial^{2}}{\partial \eta \partial \zeta} V-\frac{1}{2} \alpha_{c} C_{21} I_{1} \frac{\partial^{2}}{\partial \eta \partial \zeta} V \\
& +\frac{1}{2} I_{0} C_{55} \theta_{1}-\frac{16}{9} M \alpha^{2} I_{p 6} \alpha_{c}^{2} \frac{\partial^{2}}{\partial \zeta^{2}} \theta_{1} \\
& -M \alpha^{2} I_{p 2} \beta_{c} \alpha_{c} \frac{\partial^{2}}{\partial \eta \partial \zeta} \theta_{2}+\frac{8}{3} M \alpha^{2} I_{p 4} \alpha_{c}^{2} \frac{\partial^{2}}{\partial \zeta^{2}} \theta_{1} \\
& -\frac{16}{9} M \alpha^{2} I_{p 6} \beta_{c} \alpha_{c} \frac{\partial^{2}}{\partial \eta \partial \zeta} \theta_{2} \\
& -\frac{16}{9} M \alpha^{2} I_{p 6} \alpha_{c}{ }^{3} \frac{\partial^{3}}{\partial \zeta^{3}} W+\frac{4}{3} M \alpha^{2} I_{p 4} \alpha_{c}{ }^{3} \frac{\partial^{3}}{\partial \zeta^{3}} W \\
& +\frac{8}{3} M \alpha^{2} I_{p 4} \beta_{c} \alpha_{c} \frac{\partial^{2}}{\partial \eta \partial \zeta} \theta_{2} \\
& +\frac{4}{3} M \alpha^{2} I_{p 4} \beta_{c}^{2} \alpha_{c} \frac{\partial^{3}}{\partial \eta^{2} \partial \zeta} W \\
& -M \alpha^{2} I_{p 2} \alpha_{c}{ }^{2} \frac{\partial^{2}}{\partial \zeta^{2}} \theta_{1}+I_{1} \frac{\partial^{2}}{\partial \tau^{2}} U-\frac{4}{3} I_{3} \frac{\partial^{2}}{\partial \tau^{2}} U \\
& +\alpha_{c}^{2} I_{2} \frac{\partial^{2}}{\partial \tau^{2}} \theta_{1}-\frac{8}{3} \alpha_{c}^{2} I_{4} \frac{\partial^{2}}{\partial \tau^{2}} \theta_{1} \\
& -\frac{4}{3} \alpha_{c}^{3} I_{4} \frac{\partial^{3}}{\partial \tau^{2} \partial \zeta} W+\frac{16}{9} \alpha_{c}^{2} I_{6} \frac{\partial^{2}}{\partial \tau^{2}} \theta_{1} \\
& +\frac{16}{9} \alpha_{c}^{3} I_{6} \frac{\partial^{3}}{\partial \tau^{2} \partial \zeta} W=0 \\
& \delta \theta_{2}=\frac{4}{3} \beta_{c} \alpha_{c} C_{12} I_{4} \frac{\partial^{2}}{\partial \eta \partial \zeta} \theta_{1}+\frac{2}{3} \alpha_{c}^{2} \beta_{c} C_{21} I_{4} \frac{\partial^{3}}{\partial \eta \partial \zeta^{2}} W \\
& -\frac{8}{9} \beta_{c} \alpha_{c} C_{44} I_{6} \frac{\partial^{2}}{\partial \eta \partial \zeta} \theta_{1}+\frac{2}{3 \gamma} \alpha_{c} C_{44} I_{3} \frac{\partial^{2}}{\partial \zeta^{2}} V \\
& +\frac{4}{3} \beta_{c} \alpha_{c} C_{44} I_{4} \frac{\partial^{2}}{\partial \eta \partial \zeta} \theta_{1}-\frac{8}{9} \beta_{c} \alpha_{c} C_{12} I_{6} \frac{\partial^{2}}{\partial \eta \partial \zeta} \theta_{1} \\
& -\frac{8}{9} \alpha_{c}^{2} \beta_{c} C_{21} I_{6} \frac{\partial^{3}}{\partial \eta \partial \zeta^{2}} W \\
& +\frac{4}{3} \beta_{c} \alpha_{c} C_{21} I_{4} \frac{\partial^{2}}{\partial \eta \partial \zeta} \theta_{1}-\frac{8}{9} \beta_{c} \alpha_{c} C_{21} I_{6} \frac{\partial^{2}}{\partial \eta \partial \zeta} \theta_{1} \\
& -\frac{1}{2} \beta_{c} \alpha_{c} C_{12} I_{2} \frac{\partial^{2}}{\partial \eta \partial \zeta} \theta_{1}-\frac{1}{2} \beta_{c} \alpha_{c} C_{21} I_{2} \frac{\partial^{2}}{\partial \eta \partial \zeta} \theta_{1} \\
& -\frac{16}{9} \alpha_{c}^{2} \beta_{c} C_{44} I_{6} \frac{\partial^{3}}{\partial \eta \partial \zeta^{2}} W \\
& -\frac{8}{9} \alpha_{c}^{2} \beta_{c} C_{12} I_{6} \frac{\partial^{3}}{\partial \eta \partial \zeta^{2}} W \\
& +\frac{4}{3} \alpha_{c}^{2} \beta_{c} C_{44} I_{4} \frac{\partial^{3}}{\partial \eta \partial \zeta^{2}} W \\
& +\frac{2}{3} \alpha_{c}^{2} \beta_{c} C_{12} I_{4} \frac{\partial^{3}}{\partial \eta \partial \zeta^{2}} W \\
& -\frac{1}{2} \beta_{c} \alpha_{c} C_{44} I_{2} \frac{\partial^{2}}{\partial \eta \partial \zeta} \theta_{1}-\frac{1}{2 \gamma} \alpha_{c} C_{44} I_{1} \frac{\partial^{2}}{\partial \zeta^{2}} V \\
& -\beta_{c} C_{22} I_{1} \frac{\partial^{2}}{\partial \eta^{2}} V-\beta_{c}^{2} C_{22} I_{2} \frac{\partial^{2}}{\partial \eta^{2}} \theta_{2}
\end{aligned}
$$


$+\frac{2}{3} \beta_{c} C_{21} I_{3} \frac{\partial^{2}}{\partial \eta \partial \zeta} U-\frac{16}{9} \beta_{c}{ }^{3} C_{22} I_{6} \frac{\partial^{3}}{\partial \eta^{3}} W$

$+\frac{2}{3} \beta_{c} C_{44} I_{3} \frac{\partial^{2}}{\partial \eta \partial \zeta} U+\frac{4}{3} \beta_{c}^{3} C_{22} I_{4} \frac{\partial^{3}}{\partial \eta^{3}} W$

$-\frac{1}{2} \beta_{c} C_{12} I_{1} \frac{\partial^{2}}{\partial \eta \partial \zeta} U-\frac{1}{2} \beta_{c} C_{21} I_{1} \frac{\partial^{2}}{\partial \eta \partial \zeta} U$

$-\frac{1}{2} \beta_{c} C_{44} I_{1} \frac{\partial^{2}}{\partial \eta \partial \zeta} U-\frac{1}{2} \alpha_{c}^{2} C_{44} I_{2} \frac{\partial^{2}}{\partial \zeta^{2}} \theta_{2}$

$+\frac{8}{3} \beta_{c}{ }^{2} C_{22} I_{2} \frac{\partial^{2}}{\partial \eta^{2}} \theta_{2}-\frac{16}{9} \beta_{c}{ }^{2} C_{22} I_{6} \frac{\partial^{2}}{\partial \eta^{2}} \theta_{2}$

$+\frac{4}{3} \beta_{c} C_{22} I_{3} \frac{\partial^{2}}{\partial \eta^{2}} V+\frac{4}{3} \alpha_{c}^{2} C_{44} I_{4} \frac{\partial^{2}}{\partial \zeta^{2}} \theta_{2}$

$+\frac{2}{3} \beta_{c} C_{12} I_{3} \frac{\partial^{2}}{\partial \eta \partial \zeta} U-\frac{8}{9} \alpha_{c}^{2} C_{44} I_{6} \frac{\partial^{2}}{\partial \zeta^{2}} \theta_{2}$

$-4 \beta_{c} C_{66} I_{2} \frac{\partial}{\partial \eta} W+8 \beta_{c} C_{66} I_{4} \frac{\partial}{\partial \eta} W$

$+\frac{1}{2} I_{0} \beta_{c} C_{66} \frac{\partial}{\partial \eta} W-4 C_{66} I_{2} \theta_{2}+8 C_{66} I_{4} \theta_{2}$

$+\frac{1}{2} I_{0} C_{66} \theta_{2}-M \alpha^{2} I_{p 2} \beta_{c} \alpha_{c} \frac{\partial^{2}}{\partial \eta \partial \zeta} \theta_{1}$

$+\frac{8}{3} M \alpha^{2} I_{p 4} \beta_{c}{ }^{2} \frac{\partial^{2}}{\partial \eta^{2}} \theta_{2}-\frac{16}{9} M \alpha^{2} I_{p 6} \beta_{c}{ }^{2} \frac{\partial^{2}}{\partial \eta^{2}} \theta_{2}$

$-\frac{16}{9} M \alpha^{2} I_{p 6} \beta_{c} \alpha_{c} \frac{\partial^{2}}{\partial \eta \partial \zeta} \theta_{1}$

$-\frac{16}{9} M \alpha^{2} I_{p 6} \beta_{c} \alpha_{c}^{2} \frac{\partial^{3}}{\partial \eta \partial \zeta^{2}} W$

$-\frac{16}{9} M \alpha^{2} I_{p 6} \beta_{c}^{3} \frac{\partial^{3}}{\partial \eta^{3}} W$

$+\frac{4}{3} M \alpha^{2} I_{p 4} \beta_{c} \alpha_{c}^{2} \frac{\partial^{3}}{\partial \eta \partial \zeta^{2}} W$

$+\frac{8}{3} M \alpha^{2} I_{p 4} \beta_{c} \alpha_{c} \frac{\partial^{2}}{\partial \eta \partial \zeta} \theta_{1}$

$+\frac{4}{3} M \alpha^{2} I_{p 4} \beta_{c}^{3} \frac{\partial^{3}}{\partial \eta^{3}} W-M \alpha^{2} I_{p 2} \beta_{c}{ }^{2} \frac{\partial^{2}}{\partial \eta^{2}} \theta_{2}$

$+\frac{1}{\gamma} \alpha_{c} I_{1} \frac{\partial^{2}}{\partial \tau^{2}} V-\frac{4}{3 \gamma} \alpha_{c} I_{3} \frac{\partial^{2}}{\partial \tau^{2}} V+\alpha_{c}^{2} I_{2} \frac{\partial^{2}}{\partial \tau^{2}} \theta_{2}$

$-\frac{8}{3} \alpha_{c}^{2} I_{4} \frac{\partial^{2}}{\partial \tau^{2}} \theta_{2}-\frac{4}{3} \alpha_{c}^{2} \beta_{c} I_{4} \frac{\partial^{3}}{\partial \tau^{2} \partial \eta} W$

$+\frac{16}{9} \alpha_{c}^{2} I_{6} \frac{\partial^{2}}{\partial \tau^{2}} \theta_{2}+\frac{16}{9} \alpha_{c}^{2} \beta_{c} I_{6} \frac{\partial^{3}}{\partial \tau^{2} \partial \eta} W=0$ in which

$$
\begin{gathered}
I_{p d i}=\int_{-h / 2}^{h / 2} z^{i} d z ; \\
\left(I_{p d 2}=\frac{h^{3}}{12} ; I_{p d 4}=\frac{h^{5}}{80} ; I_{p d 6}=\frac{h^{7}}{448}\right) \\
\text { for } i=2,4,6 \\
I_{p d i}=0 \quad \text { for } i=1,3,5 \\
I_{d i}=\int_{-h / 2}^{h / 2} z^{i} \cdot\left\{1-e_{1} \cos \left(\left(\frac{\pi}{2 h}\right)\left(z+\frac{h}{2}\right)\right)\right\} d z ; \\
\text { for } i=0,1, \ldots, 6 .
\end{gathered}
$$

\section{Solution Procedure Using DQM}

The first (16) is used to separate the spatial and temporal distribution:

$$
\begin{aligned}
U(\zeta, \eta, \tau) & =U(\zeta, \eta) e^{\omega \tau}, \\
V(\zeta, \eta, \tau) & =V(\zeta, \eta) e^{\omega \tau}, \\
W(\zeta, \eta, \tau) & =W(\zeta, \eta) e^{\omega \tau}, \\
\theta_{1}(\zeta, \eta, \tau) & =\theta_{1}(\zeta, \eta) e^{\omega \tau}, \\
\theta_{2}(\zeta, \eta, \tau) & =\theta_{2}(\zeta, \eta) e^{\omega \tau},
\end{aligned}
$$

in which $\omega=\Omega a \sqrt{\rho} / \sqrt{E}$ is the dimensionless frequency ( $\Omega$ is the dimensional frequency). In DQM, the differential equations change into the first algebraic equations. According to what was mentioned, the partial derivations of the function $(F)$ are estimated by a specific variable at discontinuous points using a set of weighting series. It is supposed that $F$ has been a function representing $U, V, W, \theta_{1}$, and $\theta_{2}$ regarding variables $\xi$ and $\eta(0<\xi<1,0<\eta<1)$ when $N_{\xi} \times N_{\eta}$ is the grid points along these variables with the following derivation [22]:

$$
\frac{d^{n} F\left(\xi_{i}, \eta_{j}\right)}{d \xi^{n}}=\sum_{k=1}^{N_{\xi}} A_{i k}^{(n)} F\left(\xi_{k}, \eta_{j}\right) \quad n=1, \ldots, N_{\xi}-1 .
$$

In this equation, $A_{i k}^{(n)}$ are the weighting coefficients which are derived using Chebyshev polynomials for the positions of the grid points whose recursive formulae can be found in [23]. The standard form of the equation of motion $(M \ddot{X}+$ $K X=0$ ) is obtained by applying DQM and using (16) into governing (14a), (14b), (14c), (14d), and (14e) considering simply supported boundary conditions as mentioned below:

$$
\begin{aligned}
W_{i 1} & =V_{i 1}=U_{i 1}=\varphi_{y i 1}=\psi_{y i 1}=0, \\
\left.M_{x x}\right|_{i 1} & =0, \\
M_{x x} & =\int z \sigma_{x x} d z,
\end{aligned}
$$


TABLE 1: Comparison of the results.

\begin{tabular}{lcc}
\hline & $\omega_{1}$ & $\omega_{2}$ \\
\hline Bardell [24] & 19.7392 & 49.3480 \\
Wang et al. [25] & 19.7392 & 49.3453 \\
Wang and Shen [26] & 19.7384 & 49.3431 \\
Present work & 19.4250 & 49.3392 \\
\hline
\end{tabular}

TABLE 2: Mechanical properties of porous materials.

\begin{tabular}{lccc}
\hline & $Y$-foam & $G$-foam & Coustone \\
\hline$\rho\left(\mathrm{kg} / \mathrm{m}^{3}\right)$ & 353 & 348 & 1295 \\
$E(\mathrm{MPa})$ & 21 & 4.0 & 340 \\
$v$ & 0.35 & 0.35 & 0.35 \\
$\phi$ & 0.69 & 0.74 & 0.36 \\
Tennessee marble: $G=24(\mathrm{GPa}), v=0.3$ &
\end{tabular}

Tennessee marble: $G=24(\mathrm{GPa}), v=0.3$

$$
\begin{aligned}
W_{N i} & =V_{N i}=U_{N i}=\varphi_{y N i}=\psi_{y N i}=0, \\
\left.M_{x x}\right|_{1 N} & =0 .
\end{aligned}
$$

In this part, the eigenvalue problem is encountered in which the eigen-values of $[K / M]$ are introduced as dimensionless frequencies. It is worth mentioning that $M$ is the mass matrix and $K$ is the stiffness.

In order to ensure the correct results, linear frequency $\omega=\Omega a^{2} \sqrt{\rho \cdot h} / \sqrt{D}, D=E I /\left(1-v^{2}\right)$ for an isotropic square plate is compared in Table 1 for two modes. The results of the present work and other published papers have been matched well ([24-26]) and it shows that the correct solution has been used in the process of the research.

\section{Numerical Results and Discussion}

In this study, TSDT was used to work out the equations of motion of rectangular porous plate. The effects of aspect ratio, thickness ratio, boundary condition, porosity, and material properties are included in the results of the study. Detournay and Chen [17] have presented the material properties of saturated porous plate (Tennessee marble) and it is listed in Table 2. Besides, Table 2 reports on the properties of $Y$-foam, $G$-foam, and Coustone.

Figure 2 illustrates the effect of porosity on dimensionless natural frequency of porous plate. It is crystal clear that natural frequency is reduced by increasing the porosity. In fact, according to (5), the porosity affects Young modulus of porous plate.

Based on the thickness ratio, plates are classified, where $0.05<h / a<0.3$ has been determined for thick plates. Classical theory of plates is applicable to very thin and moderately thin plates, while HSDT applies to thick plates. Figure 2 also shows that increasing $\alpha$ leads to decrease in the dimensionless frequency for all values of porosity.

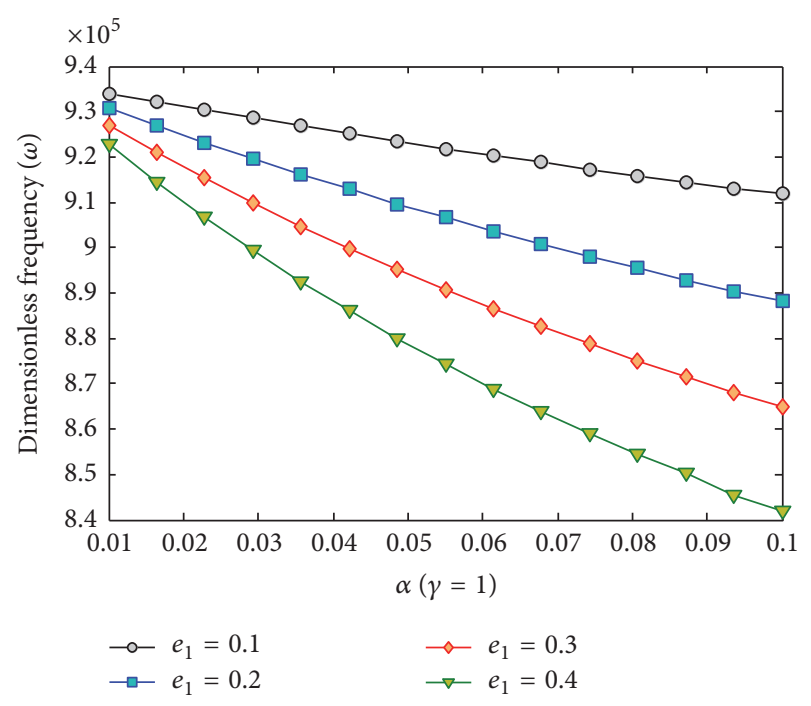

FIGURE 2: Effect of porosity and thickness ratio on dimensionless natural frequency.

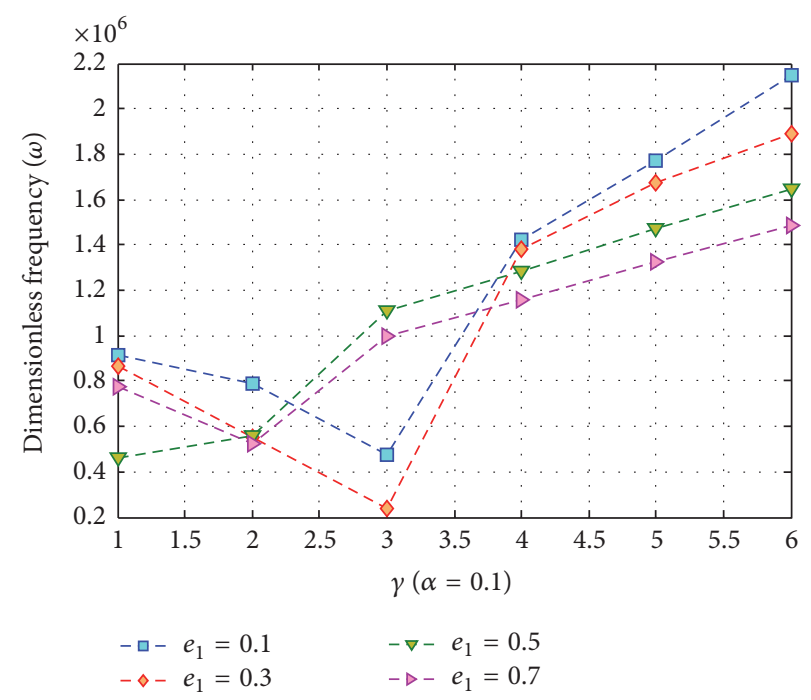

FIGURE 3: Effect of porosity and aspect ratio on dimensionless natural frequency.

Figure 3 displays the effect of aspect ratio on dimensionless natural frequency of porous plate in different porosities. Aspect ratio $(\gamma)$ is defined as a geometrical parameter of porous plate introducing the ratio of length to width. As it can be seen from Figure 3, the vibration frequency of porous plate does not necessarily follow the determined trend for $\gamma<4$ and the results are redundant in this range. But for larger aspect ratios $(\gamma \geq 4)$, the dimensionless natural frequency is increased by increasing $\gamma$.

Figures 4(a) and 4(b) show the impact of five different boundary conditions on frequency response of porous plate including clamped and simple support. Five boundary conditions of CCCC, CCCS, CCSS, CSSS, and SSSS have been compared in this figure in which the value of dimensionless 


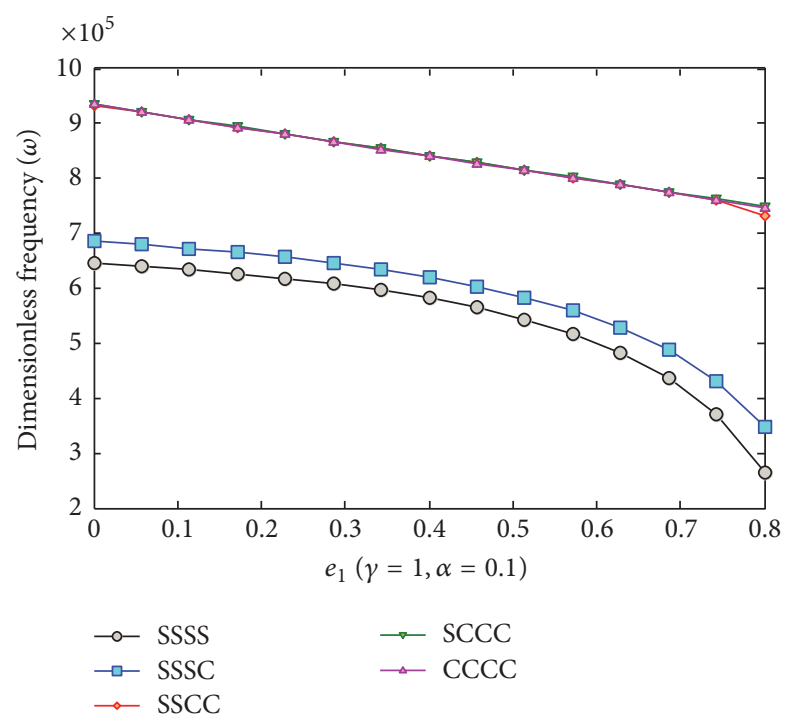

(a)

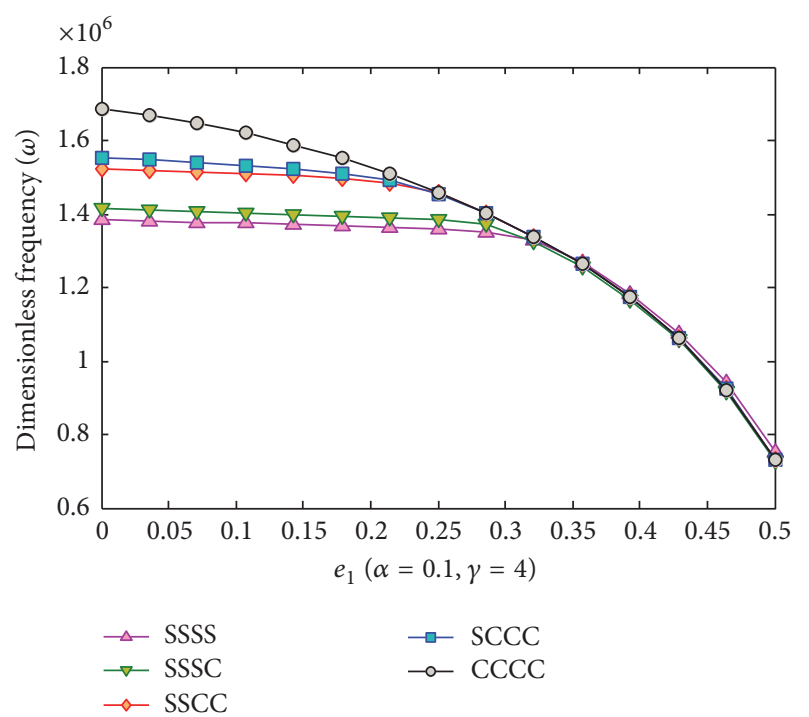

(b)

FIGURE 4: Effect of boundary conditions on dimensionless natural frequency.

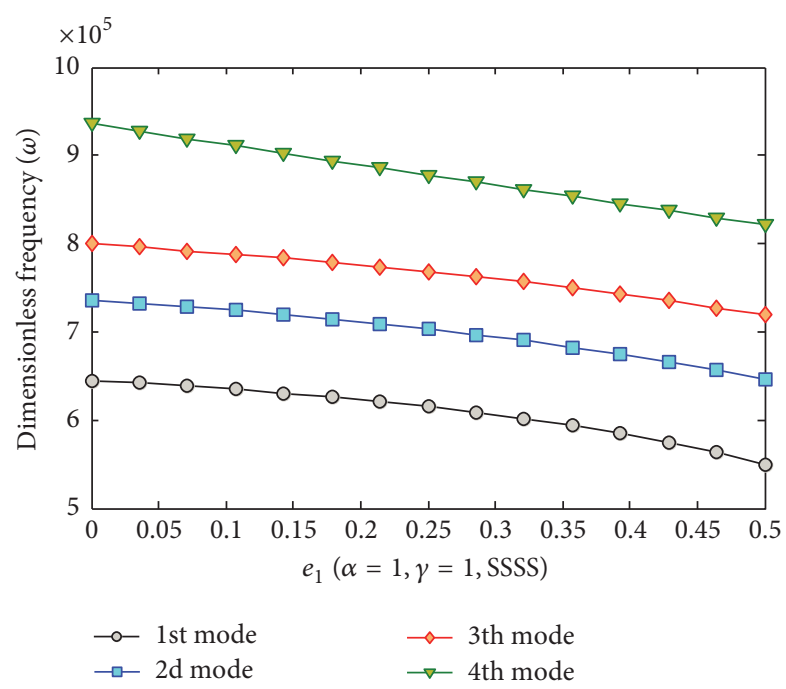

Figure 5: Variation of dimensionless natural frequency versus porosity for four primary modes.

frequency for CCCC is larger than the rest of values. Results show that CCCC boundary condition provides the maximum value for natural frequency and the minimum value is related to SSSS boundary condition. On the one hand, it can be also concluded that, in Figure 4(b) when the aspect ratio of porous plate increases, the difference among above boundary conditions decreases at a higher porosity. On the other hand, the geometrical parameters highly affect vibration behavior of porous plate.

Dimensionless frequency of porous plate versus porosity has been shown in Figure 5 for four primary modes. It is obvious from the figure that higher mode is associated with a greater frequency and also none of modes have overlap in $0.1 \leq e_{1} \leq 0.5$.
Figure 6 displays the deflection of porous plate versus porosity for four primary modes in $e_{1}=0.2, \alpha=0.2, \gamma=$ 1 , SSSS.

Figure 7 illustrates the variation of dimensionless natural frequency with porosity of porous plate in different elastic mediums. Figure 7 shows the role of elastic medium in stability of system in which the lowest curve reports the values of dimensionless frequency without elastic medium. In this figure, $\omega$ has the minimum value to the other cases. 2 th curve belongs to the Winkler foundation with spring constant $k_{w}=$ $10^{8}$. Pasternak modules create the larger values for natural frequency of porous plate due to normal and shear modules $\left(k_{w}=10^{8}, k_{g}=10^{8}\right)$.

According to Figure 8, dimensionless natural frequency of porous plate changes three materials of $Y$-foam, $G$-foam, and Coustone. $Y$-foam, $G$-foam, and Coustone are known as porous materials with different Young modulus and porosities. According to Table 1, comparisons have been made: Young modulus of Coustone is larger than foams and the porosity of foams is higher than Coustone. Among foams, $Y$ foam has a larger Young modulus and a lower porosity, so the most dimensionless natural frequency belongs to Coustone plate and the lowest one is for $G$-foam.

\section{Conclusion}

The significance and novelty of this research lie in studying free vibration of embedded porous which is a relatively new and prosperous topic in this field. The linear poroelasticity theory was used to work out the stress-strain relations in porous material while the Young modulus and density vary in transverse direction of porous plate. To evaluate the effect of normal and shear modulus on the stability of porous plate, Pasternak foundation was developed. TSDT with five unknown functions was used to derive the equation of 

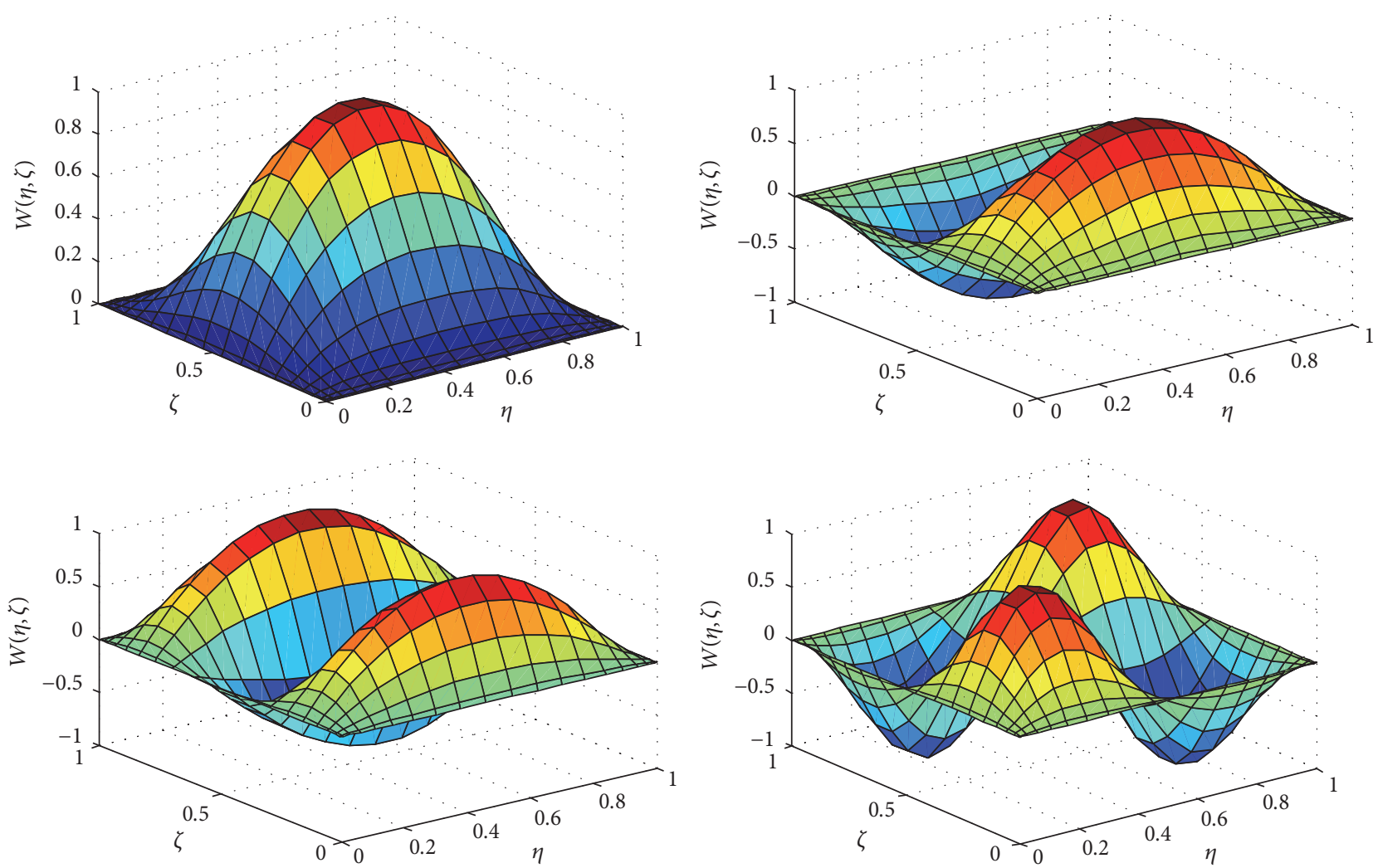

FIGURE 6: Deflection of porous plate versus porosity for four primary modes in $\left(e_{1}=0.2, \alpha=0.2, \gamma=1\right.$, SSSS).

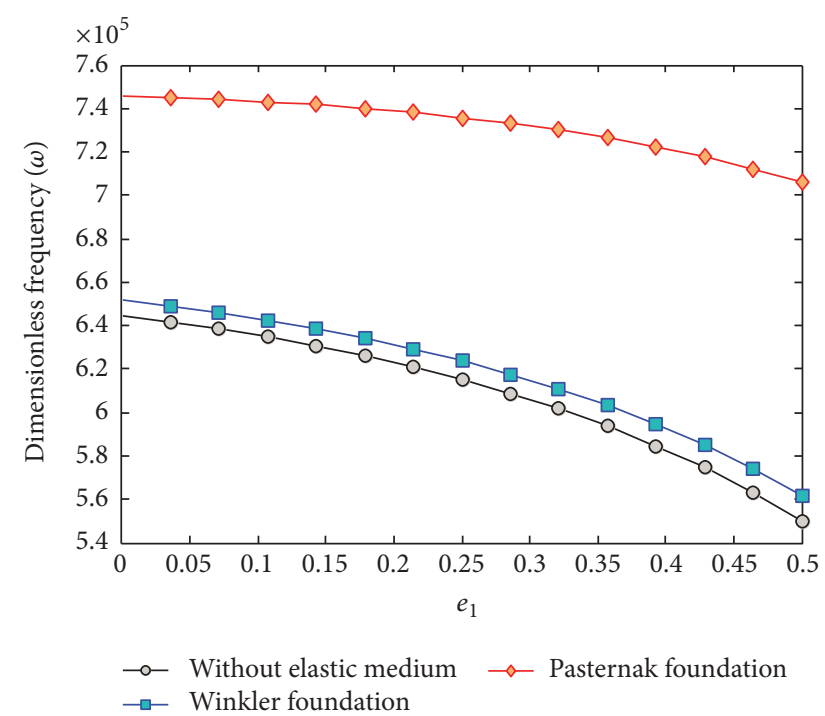

FIGURE 7: Effect of elastic medium on dimensionless natural frequency.

motions. A set of equations were solved by two-dimensional DQM and the following results were concluded:

(i) Regarding the relationship between porosity, Young modulus, and density in porous materials, the high porosity leads to reduction of dimensionless

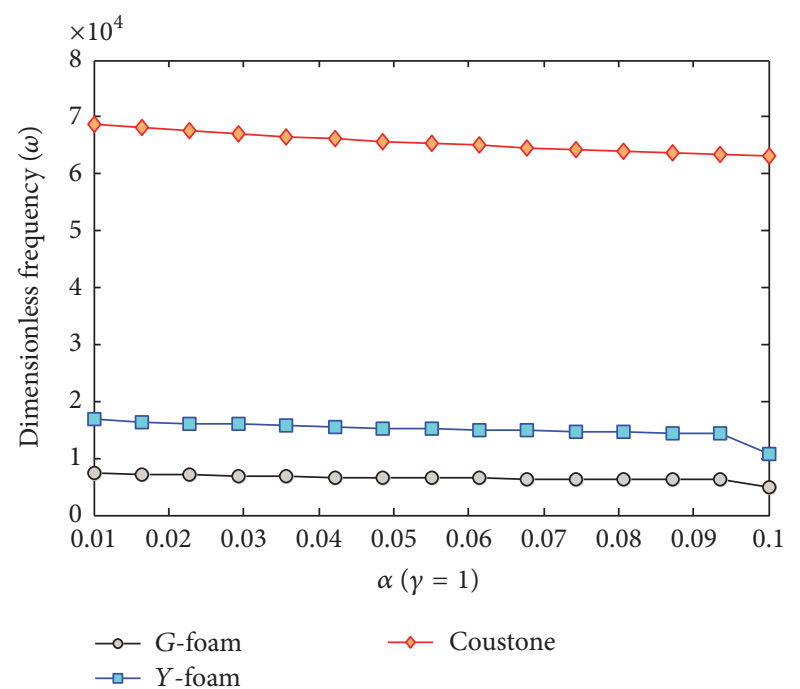

FIGURE 8: Effect of material properties on dimensionless natural frequency.

frequency of porous plate. On the other hand, porosity reduces the density and Young modulus of matter. Thus, porous materials have the lower natural frequency due to porosity. The porosity in porous materials also creates the damping that it can be used in the aerospace industry. 
(ii) Pasternak with normal $\left(k_{b w}\right)$ and shear $\left(K_{b g}\right)$ modulus can be regarded as important factors in stability of porous plate in which the dimensionless frequency increases with increasing the stiffness. So the vibration frequency of porous plate can be increased by providing a hard bed.

(iii) Aspect ratio and thickness ratio as geometrical parameters have an efficient and vital role in vibration behavior of porous plate. Therefore, choosing the right dimensions and thickness can be achieved to a certain natural frequency.

(iv) Result shed light on the ground that CCCC boundary condition provides maximum value for natural frequency of porous plate and minimum value relates to SSSS boundary condition. It is evident that CCCC boundary condition increases the stiffness of porous plate.

(v) Coustone as a porous material has larger natural frequency in comparison to $Y$-foam and $G$-foam due to high Young modulus and density.

The result of this study can benefit the designers and manufacturers of aircrafts, cars, automobiles, and pneumatic conveying and material handling systems including aerators, airlocks, bins, blenders, blowers, boosters, control systems, air and gas compressors, ship, and barge loaders and unloaders.

\section{Competing Interests}

The authors declare that they have no competing interests.

\section{Acknowledgments}

This work was supported by University of Kashan [Grant no. 574600/14].

\section{References}

[1] Porous Materials, http://www.uio.no/studier/emner/matnat/ kjemi/KJM5100/h06/undervisningsmateriale/16KJM5100_2006_ porous_e.pdf.

[2] M. A. Biot and D. G. Willis, "The elastic coefficients of the theory of consolidation," Journal of Applied Mechanics, vol. 24, pp. 594-601, 1957.

[3] D. Mansutti, G. Pontrelli, and K. R. Rajagopal, "Steady flows of non-Newtonian fluids past a porous plate with suction or injection," International Journal for Numerical Methods in Fluids, vol. 17, no. 11, pp. 927-941, 1993.

[4] P. Leclaire, K. V. Horoshenkov, M. J. Swift, and D. C. Hothersall, "The vibrational response of a clamped rectangular porous plate," Journal of Sound and Vibration, vol. 247, no. 1, pp. 19-31, 2001.

[5] P. Leclaire, K. V. Horoshenkov, and A. Cummings, "Transverse vibrations of a thin rectangular porous plate saturated by a fluid," Journal of Sound and Vibration, vol. 247, no. 1, pp. 1-18, 2001.

[6] M. Schanz and S. Diebels, "A comparative study of Biot's theory and the linear theory of porous media for wave propagation problems," Acta Mechanica, vol. 161, no. 3, pp. 213-235, 2003.
[7] D. Debowski and K. Magnucki, "Dynamic stability of a porous rectangular plate," Proceedings in Applied Mathematics and Mechanics, vol. 6, no. 1, pp. 215-216, 2006.

[8] A. R. Khorshidvand, E. F. Joubaneh, M. Jabbari, and M. R. Eslami, "Buckling analysis of a porous circular plate with piezoelectric sensor-actuator layers under uniform radial compression," Acta Mechanica, vol. 225, no. 1, pp. 179-193, 2014.

[9] M. Jabbari, A. Mojahedin, A. R. Khorshidvand, and M. R. Eslami, "Buckling analysis of a functionally graded thin circular plate made of saturated porous materials," Journal of Engineering Mechanics, vol. 140, no. 2, pp. 287-295, 2014.

[10] M. Jabbari, A. Mojahedin, and M. Haghi, "Buckling analysis of thin circular FG plates made of saturated porous-soft ferromagnetic materials in transverse magnetic field," Thin-Walled Structures, vol. 85, pp. 50-56, 2014.

[11] E. F. Joubaneh, A. Mojahedin, A. R. Khorshidvand, and M. Jabbari, "Thermal buckling analysis of porous circular plate with piezoelectric sensor-actuator layers under uniform thermal load," Journal of Sandwich Structures and Materials, vol. 17, no. 1, pp. 3-25, 2015.

[12] A. Behravan Rad and M. Shariyat, "Three-dimensional magneto-elastic analysis of asymmetric variable thickness porous FGM circular plates with non-uniform tractions and Kerr elastic foundations," Composite Structures, vol. 125, pp. 558-574, 2015.

[13] F. Ebrahimi and A. Jafari, "A Higher-order thermomechanical vibration analysis of temperature-dependent FGM beams with porosities," Journal of Engineering, vol. 2016, pp. 1-20, 2016.

[14] M. Bourada, A. Kaci, M. S. A. Houari, and A. Tounsi, "A new simple shear and normal deformations theory for functionally graded beams," Steel and Composite Structures, vol. 18, no. 2, pp. 409-423, 2015.

[15] A. M. A. Neves, A. J. M. Ferreira, E. Carrera et al., "A quasi3D hyperbolic shear deformation theory for the static and free vibration analysis of functionally graded plates," Composite Structures, vol. 94, no. 5, pp. 1814-1825, 2012.

[16] H. Hebali, A. Tounsi, M. S. A. Houari, A. Bessaim, and E. A. A. Bedia, "New quasi-3D hyperbolic shear deformation theory for the static and free vibration analysis of functionally graded plates," Journal of Engineering Mechanics, vol. 140, no. 2, pp. 374383, 2014.

[17] E. Detournay and A. H. D. Chen, Fundamentals of Poroelasticity, Pergamon Press, 1993.

[18] J. N. Reddy, Theory And Analysis of Elastic Plates And Shells, CRC Press, New York, NY, USA, 2007.

[19] C. M. Wang, J. N. Reddy, and K. H. Lee, Shear Deformable Beam and Plate Relation with Classical Solution, Elsevier Science, London, UK, 2000.

[20] J. N. Reddy, Energy Principles and Variational Methods in Applied Mechanics, John Wiley \& Sons, Hoboken, NJ, USA, 2000.

[21] A. Ghorbanpour Arani and Z. Khoddami Maraghi, "A feedback control system for vibration of magnetostrictive plate subjected to follower force using sinusoidal shear deformation theory," Ain Shams Engineering Journal, vol. 7, no. 1, pp. 361-369, 2014.

[22] A. Ghorbanpour Arani, Z. Khoddami Maraghi, and H. Khani Arani, "Orthotropic patterns of Pasternak foundation in smart vibration analysis of magnetostrictive nanoplate," Journal of Mechanical Engineering Science: Part C, vol. 230, no. 4, pp. 559572, 2015.

[23] C. Shu, Differential quadrature and its application in engineering, Springer, London, UK, 2000. 
[24] N. S. Bardell, "The application of symbolic computing to the hierarchical finite element method," International Journal for Numerical Methods in Engineering, vol. 28, no. 5, pp. 1181-1204, 1989.

[25] Y. Wang, X. Wang, and Y. Zhou, "Static and free vibration analyses of rectangular plates by the new version of the differential quadrature element method," International Journal for Numerical Methods in Engineering, vol. 59, no. 9, pp. 12071226, 2004.

[26] Z.-X. Wang and H.-S. Shen, "Nonlinear vibration and bending of sandwich plates with nanotube-reinforced composite face sheets," Composites Part B: Engineering, vol. 43, no. 2, pp. 411421, 2012. 


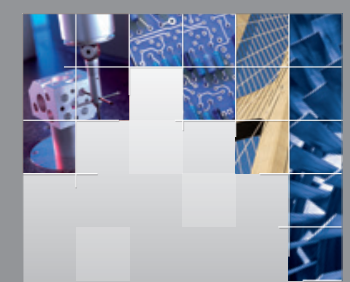

\section{Enfincering}
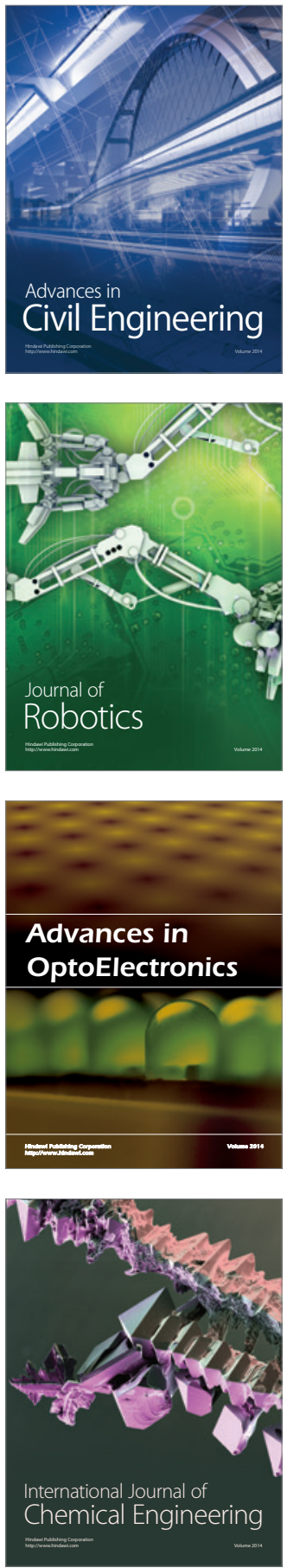

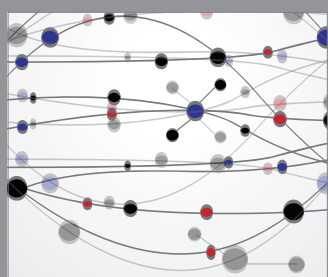

The Scientific World Journal

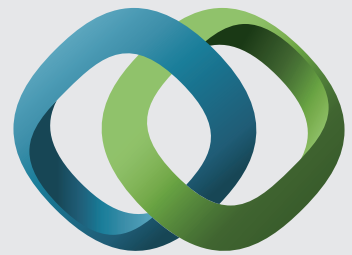

\section{Hindawi}

Submit your manuscripts at

https://www.hindawi.com
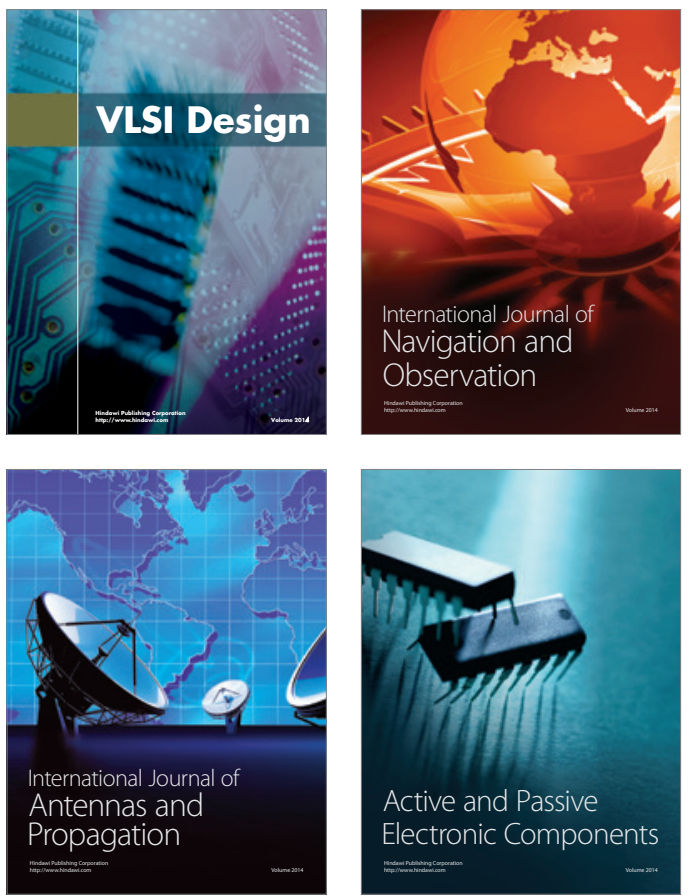
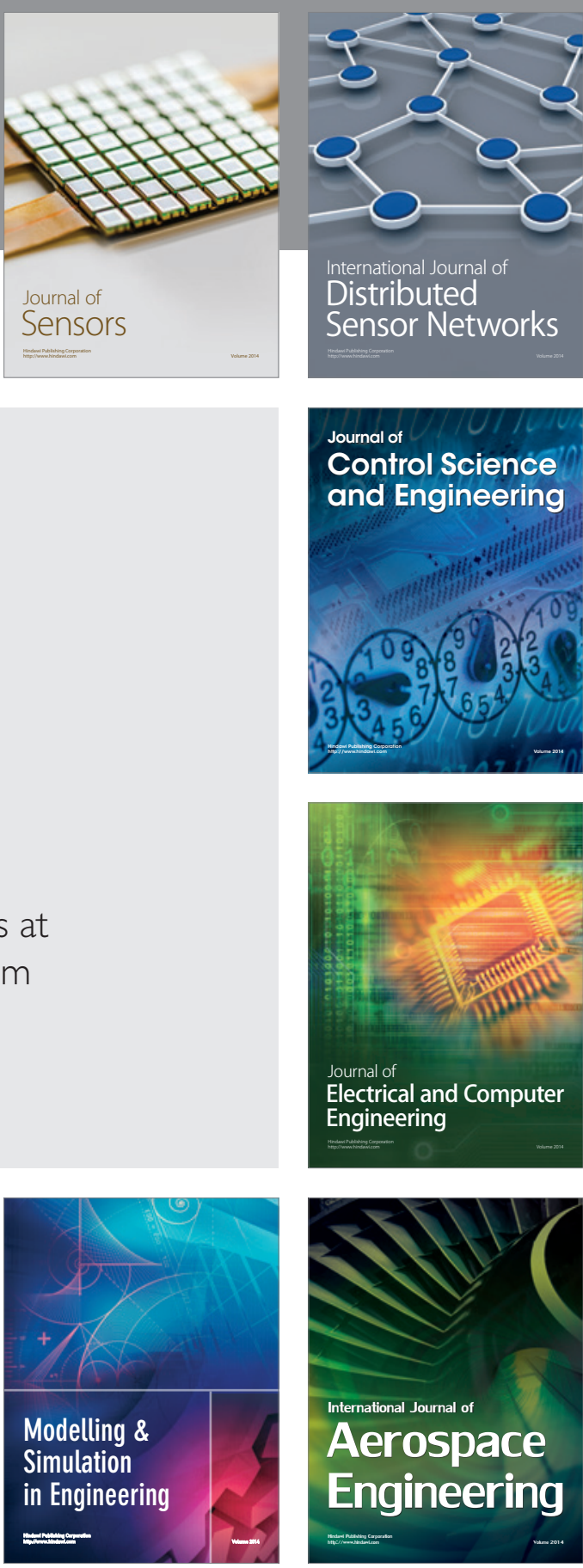

International Journal of

Distributed

Sensor Networks

$-$

Joumal of

Control Science

and Engineering
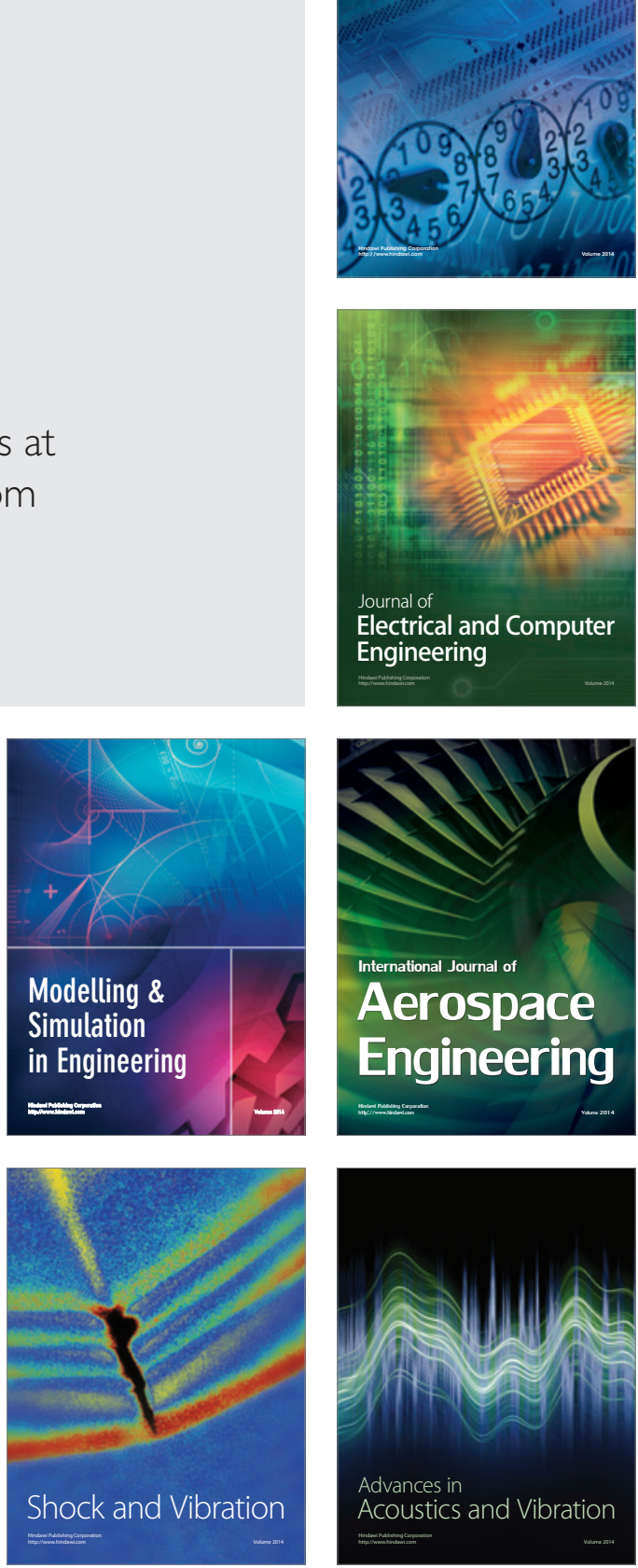